\title{
Food Neophobia in Childhood
}

\section{Camille Rioux ${ }^{1}$,}

Author Affiliations:

${ }^{1}$ Max Planck Institute for Human Development, Max Planck Research Group Naturalistic Social Cognition, Lentzeallee 94, 14195 Berlin, Germany.

Correspondence should be addressed to: Camille Rioux, Max Planck Institute for Human Development, Max Planck Research Group Naturalistic Social Cognition, Lentzeallee 94, 14195 Berlin, Germany. Email: rioux@mpib-berlin.mpg.de

This book chapter is published in the Handbook of Eating and Drinking

Rioux C. (2019) Food Neophobia in Childhood. In: Meiselman H. (eds) Handbook of Eating and Drinking. Springer, Cham. https://doi.org/10.1007/978-3-319-75388-1_159-1 


\begin{abstract}
Food neophobia, is defined as the reluctance to eat new foods. It has been presented as one of the main obstacles to improving children's intake of fruits and vegetables. Hence, it is essential to uncover the factors that influence the development or expression of food neophobia in childhood, in order to promote the adoption of healthy eating behaviors early in life. To date, research on neophobia has revealed a large range of factors influencing this disposition: both individual factors (e.g., genetic influences and temperament traits) and environmental factors (e.g., early food experiences and feeding practices). Various strategies can efficiently decrease children's food neophobia expression, such as taste and visual exposure to new foods. Further studies could systematically evaluate the effectiveness of such interventions, as well as investigate the mechanisms behind positive effects.
\end{abstract}

\title{
1. Introduction
}

Children's low consumption of fruits and vegetables is a common source of concern for parents who are worried about their child's dietary diversity and quality (DeCosta et al. 2017). Food neophobia, defined as the reluctance to eat new foods, has been presented as one of the main obstacles to improving children's intake of fruits and vegetables (Cole 2017; Dovey et al. 2008; Lafraire et al. 2016). The impact of food neophobia extends well beyond childhood, since dietary habits acquired during childhood largely determine dietary patterns in adulthood (Nicklaus et al. 2005). It is therefore essential to understand the mechanisms underpinning food neophobia in childhood, in order to promote the adoption of healthy eating behaviors early in life. This chapter aims to examine the question of food neophobia in typically developing children (adults, and those children who have been diagnosed with a pediatric disorder such as Avoidant/Restrictive Food Intake Disorder(ARFID) are out of the scope of the present chapter). The chapter starts by discussing how to characterize food neophobia and a general approach to it's measurement. The second part provides an overview of the factors influencing food neophobia. Then, an evaluation of the effectiveness of different interventions aimed at reducing food neophobia is presented, highlighting on interventions based on food exposure, and further opportunities for research and interventions are explored.

\section{Characterization and measurement of food neophobia}

\subsection{Definition}


Food neophobia is defined as the reluctance to eat new foods, or the fear of new foods (Pliner \& Hobden 1992). Food neophobia can be considered as a true phobia (Maratos \& Sharpe 2018): it is associated with typical physiological fear responses, such as galvanic skin response (i.e., changes in sweat skin activity) and an increase in pulse or respiration rhythm. Additionally, food neophobia in children is often associated with an increased anxiety or even disgust over new foods, as well as with attentional biases toward new foods. All three components (anxiety, disgust, or attentional biases) are often found in phobias (see Maratos \& Sharpe 2018 for a review of food neophobia as a true phobia). This fear of new food is assumed to serve a protective function for omnivorous species (i.e., species that do not eat just one type of food) in potentially hostile food environments (Pliner \& Hobden 1992; Rozin 1979). Rozin (1979) described food neophobia as an efficient adaptive strategy for children to avoid the risk of ingesting new (and thus potentially poisonous) food items, once they are mobile enough to reach for, and consume food available in their proximal environment. Some authors have suggested that this view explains why plant-based foods such as fruits and vegetables are the privileged targets of food neophobia as plants often contain secondary coumpounds that can be toxic to human and no visual features reliably signal which plants may contain these toxic compounds (Cashdan 1998; Wertz \& Wynn 2014; Włodarczyk et al. 2018).

The notion of novelty plays a central role in the definition of food neophobia. This notion has to be broad since a rejected new food can be (i) a food item the child has never encountered before, (ii) a food that has been encountered before but, due to variations in color, shape, preparation, etc., is not currently recognized by the child. This means that new foods can include items previously accepted by the child, well known from the caregivers' perspective, and frequently consumed in the cultural area the child belongs to (e.g., a tomato for most European children). In other words, it is from the child's perspective that the notion of novelty has to be analyzed. A new food item can be genuinely new (e.g., a dragon fruit for most European children) or perceived as new (e.g., a tomato cut into cubes instead of slices). Some authors have suggested that this view also explains why fruits and vegetables are the privileged targets of food neophobia as they are more likely to exhibit changes in color, shape or preparation between servings than other foods (e.g., starchy foods) and therefore can be more easily perceived as new by children, even though they have been encountered in other forms before (Harris 2018). 
In both cases (genuinely new food or food perceived as new) the presented food is not subsumed under a known category (e.g., the category tomato), and properties of the food cannot be inferred (such as the property edible if the presented food would be categorized as a tomato). Categorization is a fundamental cognitive process that allows us to organize objects into groups that share common characteristics (Murphy 2002). One main use of categories is to make predictions about new items (e.g. tomatoes are edible, therefore if a new item is categorized as a tomato one can infer that this new item is edible) (Murphy 2002). Accordingly, some researchers suggest that food neophobia is closely intertwined with the development of categorization abilities in the food domain, abilities that largely improve around 2- to 3-years of age as children become more mobile (Harris 2018; Lafraire et al. 2016; Nicklaus \& MonneryPatris 2018; Rioux et al. 2016; 2017b). More precisely, Rioux and colleagues recently found a robust association between food neophobia and food categorization abilities in 2- to 6-year-old children (Rioux et al. 2016; 2017b). Categorical judgments are mainly made based on visual cues as neophobic reaction results in new foods being rejected on mere sight, even before being tasted or swallowed (Cashdan1998; Harris 2018; Dovey et al. 2008; Lafraire et al. 2016). Vision is indeed the main sensory component predicting whether a child would try a new food (Nicklaus \& Monnery-Patris 2018).

\subsection{Trait neophobia vs. state neophobia}

Food neophobia is most often conceptualized as a personality trait, a continuum along which children (and more generally individuals) can be located in terms of their stable and persistent propensity to avoid new foods (Alley 2018; Dovey et al. 2008; Pliner \& Hobden 1992). Indeed, as listed below, food neophobia exhibits most of the characteristics known as supporting the definition of a personality trait:

(a) Heritability. For instance, comparing food neophobia between monozygotic twins (sharing all their genes) and dizygotic twins (sharing on average half their genes), researchers found that food neophobia is highly heritable (see section 3.1.1 below).

(b) Linked to biological mechanisms (e.g., neurochemical, neuroanatomical). A handful of studies have reavealed implication of neural mechanisms in food neophobia (Klarer et al. 2014).

(c) Early ontogenetic appearance. Food neophobia appears in the first few years of life and is fully expressed by preschool age (see section 2.4 below).

(d) Counterparts exist in non-human primates as well as in certain other social mammals. 
Neophobic behaviors are commonly observed among omnivorous species, such as capuchin monkeys or rats (Rozin 1979).

(e) Generation of important individual differences. Pliner and Hobden (1992) developed

a scale to assess the level of individual food neophobia and found a great interindividual variation in respondants' answers: some respondants obtained low scores and could be defined as neophilic while other obtained high scores and could be defined as neophobic.

Nevertheless, food neophobia does not seem to exhibit one important characteristic of a personality trait: to be relatively enduring and predictive of conceptually coherent outcomes. Indeed, while neophobia is thought to be relatively stable during early childhood, most researchers agree that, in late childhood, the expression of food neophobia decreases (see section 2.4 below). Additionally, food neophobia is only partially predictive of coherent behavioral outcomes, since most studies found only medium correlations between neophobia and willingness to try new foods. A recent review of instruments developed to measure food neophobia (Damsbo-Svendsen et al. 2017) revealed that the highest correlation between neophobia and willingness to try new foods found in the literature was 0.47 . The lowest correlation was 0.11 (correlation coefficients range from -1 to 1 . These two end points mean that two constructs are perfectly negatively/postively correlated, while a coefficient of 0 means that the two constructs are not at all correlated). This led some authors to rather address neophobia as a state (Alley 2018), a transitory negative emotion (in the case of neophobia, fear), elicited by the idea of ingesting a specific food, in a specific context. In this view, the expression of food neophobia can be unstable, age-dependent, and modulated by context modalities such as food properties, individual's mood, or social modeling (Alley 2018).

To reconcile the distinction between food neophobia as a trait or as a state the concept of disposition is enlightening (Mumford 1998). Dispositions are seen as genuine and functional properties ascribed to objects, kind or persons (e.g., the disposition fragile for a glass). To each dispositional property corresponds a typical manifestation (e.g., shatter when struck for a fragile glass), explained by a physical/biological state or mechanism (possibly unknown). Importantly, to possess a disposition is not to be in a particular state, but to be liable to be in a particular state when certain circumstances are met (e.g., if the fragile glass is struck, it shatters). Neophobia could then be viewed as a disposition. Only when neophobic children are presented with new foods, they will present rejection behaviors. The circumstances are met when a food is 
genuinely new or perceived as new. As children develop and get older, fewer things are new for them, thus the circumstances that trigger food rejection behaviors occur more and more rarely, potentially explaining the non-stability of neophobia during the lifespan.

\subsection{Measurement of food neophobia in childhood}

\subsubsection{Questionnaires}

Methodologically speaking, it appears that when neophobia is conceptualized as a trait, questionnaires are used (Alley 2018). This is due in large part to ease and rapidity of administering such measures (Alley 2018). The first researchers to develop a food neophobia scale were Pliner and Hobden (1992), who designed the Food Neophobia Scale (FNS). This questionnaire asks adult participants to indicate the extent to which they agree, or not, with statements about consumption of new foods, such as "If I don't know what is in a food, I won't try it" (see Fig. 1). Originally devised to score adults' neophobia, the FNS was rapidly adapted to measure children's neophobia (e.g., "If my child does not know what is in a food, she/he won't try it", Children Food Neophobia Scale, CFNS; Pliner 1994). This scale has been widely used to measure food neophobia in childhood and successfully adapted (e.g., the Fruits and Vegetables Neophobia Instrument, FVNI, the Food Neophobia Test Tool, FNTT, the Questionnaire pour Enfant de Neophobie Alimentaire, QENA, see Damsbo-Svendsen et al. 2017 for a recent review on tools to measure food neophobia). It has also been translated into several languages, including for instance French, Spanish, Chinese, Swedish and Italian (Damsbo-Svendsen et al. 2017).

Figure 1: Items of the original Food Neophobia Scale (retrieved from Pliner, 1994).

\footnotetext{
I am constantly sampling new and different foods.

I don't trust new foods.

If I don't know what is in a food, I won't try it.

I like foods from different countries.

Ethnic food looks too weird to eat.

At dinner parties, I will try a new food.

I am afraid to eat things I have never had before.

I am very particular about the foods I will eat.

I will eat almost anything.

I like to try new ethnic restaurants.
}

Both auto-assessment scales (i.e., children respond for themselves,) and hetero-assessment scales (i.e., caregivers respond for their children) are used. Hetero-assessment scales are commonly used with children younger than 5- to 6-year-old (Alley 2018). It is important to 
have in mind that hetero-assessment scales give only approximation of children's own food behaviors. There are potential social desirability biases (i.e., parents may present a better image of their children or themselves), or parents may sometimes project their own behaviors onto those of their children (Alley, 2018). In fact, caregivers' reports of their children's neophobia only moderately correlate with children's reports of their own neophobia (e.g., $r=0.3$ in Pliner 1994). Both auto-assessment scales and hetero-assessment scales also have the potential pitfall of memory errors (Alley 2018). Additionally, recent reviews (Alley 2018; Damsbo-Svendsen et al. 2017; de Lauzon-Guillain et al. 2012) pointed out that several existing scales measuring children's food neophobia are not completely reliable, potentially leading to deceptive conclusions. For instance, the widely used FNS/CFNS, failed to validate construct validity and/or temporal reliability (de Lauzon-Guillain et al. 2012). This means that they can measure something else than neophobia (failure to validate construct validity) and provide different outcomes if completed several times (failure to validate temporal reliability). This urges caution of the choice of the scale selected to measure food neophobia in children.

\subsubsection{Willingness to try new food tasks}

Rather than asking people about consumption of new food in general, one can attempt to determine actual consumption of specific new foods (Alley 2018). There are some clear advantages, to using direct consumption measures. When faced with a request to actually select or eat new food items, young children might provide better information on the attractiveness of particular new food items, than when expected to deal with a purely verbally based assessment, as the actual presence of food is believed to trigger the emotional response. This strategy is actually often used when food neophobia is considered as a state. However, there are a multitude of factors that shape an individual's food choices and vary across time and situation (such as hunger level) and make it difficult to create a test that purely reflects variation in food neophobia (Alley 2018). These measures of food neophobia are thus better treated as a means to test the construct validity of questionnaires (Alley 2018; Damsbo-Svendsen et al. 2017).

The most common task used is the "Willingness to Try New Food" task (WTNF), in which children are presented with new foods and are asked to try them. The first WTNF task was developed by Pliner and Hobden (1992). It consists of participants ranking six a priori new foods from five categories (salads, dips, crackers, vegetables and snacks) in order of their willingness to taste them (in a fictitious subsequent tasting session). Pliner and Hobden's food task has been widely used and largely adapted (Alley 2018; Damsbo-Svendsen et al. 2017). 
Some authors use pictures of foods instead of actual foods, while others use real foods. Some authors add familiar foods to the task, and compare the number of familiar foods tasted to the number of new foods tasted. Further, in some WTNF, children have to taste the foods, while on the original task, they just had to indicate which foods they were willing to taste (see DamsboSvendsen et al 2017 for a review of WTNF tasks). Usually, the willingness to try new foods is moderately to highly correlated to scores on food neophobia scales.

\subsection{Developmental trend and gender differences}

Most researchers agree that neophobic behaviors appear at around 2 years of age, when children become more mobile and could find themselves outside parental guidance (see Dovey et al., 2008 and Lafraire et al., 2016 for reviews on the developmental pattern of food neophobia). A few recent studies have also discovered precursors of food neophobia in infancy, though after weaning (Harris 2018; Moding \& Stifter 2016). There is however a contention in the literature as to whether neophobic behaviors increase progressively thereafter, or remain stable during early childhood. On the one hand, some authors found that 5-year-old children tend to be more neophobic than their younger counterparts. On the other hand, others authors did not find any correlation between food neophobia and age within the 2- to-6-year-old age range (see Lafraire et al. 2016 for a review, see Table 1). Longitudinal studies on food neophobia are scarce and are needed to investigate further its developmental trend. Nevertheless, there is a relative consensus that in late childhood/beginning of adolescence, the expression of food neophobia decreases, until it reaches a relatively stable expression in adulthood (Dovey et al. 2008). Actually, the mere fact that fewer things are new for an adult or an adolescent than for a child, automatically reduces the expression of food neophobia (Cooke \& Wardle, 2005).

Table 1: Summary of studies relating food neophobia to age during early childhood.

\begin{tabular}{llll}
\hline Publication & Population & Measurement instrument & Correlation with age \\
\hline$\underline{\text { Addessi et al. 2005 }}$ & $\underline{\text { 2-5 years,US }}$ & $\underline{\text { WTNF task }}$ & $\underline{\text { No association }}$ \\
$\underline{\text { Barends et al., 2014 }}$ & $\underline{\text { 12-23 months, The }}$ & $\underline{\text { WTNF task }}$ & $\underline{\text { positive }}$ \\
& $\underline{\text { Netherlands }}$ & & \\
$\underline{\text { Cashdan 1994 }}$ & $\underline{\text { 1-10 years, US }}$ & $\underline{\text { questions on reaction to novel }}$ & $\underline{\text { positive }}$ \\
$\underline{\text { Cooke et al 2003 }}$ & $\underline{\text { 2-6 years,UK }}$ & $\underline{\text { CFNS }}$ & $\underline{\text { No association }}$
\end{tabular}


$\underline{\text { Koivisto-Hurti }}$ \& 2-9 years, Sweden

Sjoden 1996

$\underline{\text { Monnery-Patris et al } 20-22 \text { months }}$

Children's Eating Difficulties

No association $\underline{2015}$

Concerning potential gender differences, most studies investigating food neophobia during childhood did not find gender differences (see Dovey et al. 2008 for a review). Though, some studies have found differences in both directions (i.e., girls being more neophobic than boys and vice versa, see Dovey et al. 2008), however, overall there is still limited evidence for a robust association between food neophobia and gender (Dovey et al. 2008).

\subsection{Food neophobia and its association with diet quality and weight status}

Food neophobia has been shown to affect children's diet and food preferences. The food items that are rejected the most are certainly fruits and vegetables (see Kral 2018, Cole 2017 and Nicklaus \& Monnery-Patris 2015 for reviews on food neophobia and its association with diet quality and weight status). Numerous studies have indeed uncovered negative associations between food neophobia and intake of fruits and vegetables (Cole et al. 2017, Nicklaus \& Monnery-Patris 2015, see Table 2). This often goes along with a negative association between food neophobia and liking for these food products (Henriques et al. 2009; Laureati et al. 2015). Besides fruits and vegetables, the intake of other food groups such as meat and protein are also negatively impacted by food neophobia (Kral 2018). Therefore, food neophobia in children is associated with decreased dietary variety and quality (Kral 2018) along with a possible lack of essential micro-nutriments and fibers that are necessary for a normal and healthy child development (Dovey et al. 2008; Lafraire et al. 2016).

Table 2: Summary of studies relating food neophobia to dietary intake outcomes (adapted from Monnery-Patris et al 2015).

\begin{tabular}{llll}
\hline Publication & Population & Measurement instrument & $\begin{array}{l}\text { Dietary intake outcomes in } \\
\text { neophobic children }\end{array}$ \\
\hline Cooke et al. 2003 & 2-6 years, UK & CFNS (only 6 items) & $\begin{array}{l}\text { Lower consumption of fruits, } \\
\text { vegetables, meat and egg. }\end{array}$
\end{tabular}


Cooke et al. 2006

4-5 years, UK

CFNS (only 6 items)

Galloway et al. 7 years, US

CFNS

2003

Helland et al 201

2-3 years, Finland

CFNS

Maiz \& Balluerka 8-16 years

CFNS

2016

Nicklause et al. 4-22 years 2005

Perry et al $2005 \quad 24$ months

CFNS
Lower consumption of fruits, vegetables, meat and egg.

Lower consumption of vegetables .

Lower consumption of fruits, vegetables, and fish.

Lower consumption of fruits, vegetables, and fish. Higher consumption of sweets and candy.

Lower consumption of all food groups.

Lower consumption of fruits and vegetable. Higher consumption of discretionary foods.

Regarding weight status, studies that have systematically examined the relationship between food neophobia and BMI scores are scarce and have produced mixed results. Some studies found no association between BMI and food neophobia, but other found that neophobic children tend to be overweight, as they seem to eat few fruits and vegetables and high quantities of discretionary foods, Cole et al. 2017). Overall there is limited evidence to date for a robust association between food neophobia and BMI scores in children and adolescents (Kral, 2018).

\subsection{Food neophobia and its relationship with food pickiness}

Food neophobia is often associated with food pickiness, another type of food rejection (neophobic children are often also picky and vice versa) (Dovey et al. 2008). While food neophobia is well defined, the definition of food pickiness is more imprecise and a matter of contention. Generally, it is defined as the rejection of new and previously accepted foods, along with the rejection of certain textures that are rejected after the tasting step (i.e., foods are tasted but not swallowed) (Dovey et al. 2008; Lafraire et al. 2016; Rioux et al. 2017a). Rioux and 
colleagues have recently suggested that theoretically speaking, food neophobia and pickiness may capture a similar kind of fear for new and potentially toxic foods. Indeed, as with food neophobia, pickiness presents at least one signature feature of a phobia, that is to say an increased disgust over food items in general (Rioux et al. 2017a). Moreover, texture rejection, an important component of food pickiness, can prevents children from the ingestion of decayed food that can potentially become toxic foods (as texture can inform of the decay of a food). Additionally, the authors suggested that what is thought to be rejection of familiar foods is probably rejection of foods perceived as new for children (see section 2.1). Nevertheless, neophobia and pickiness appear to be two behaviorally distinct kinds of food rejection (Dovey et al., 2008; Lafraire et al., 2016; Rioux et al., 2017a). Although the motivation for rejection may be the same, caregivers appear to distinguish food neophobia from pickiness: distinguishing rejection due to texture (pickiness) from rejection due to sight (neophobia), and rejection after the tasting step (pickiness) from rejection before the tasting step (neophobia).

\section{Typology of factors influencing food neophobia}

Extensive research on food neophobia has revealed a large range of factors influencing this disposition in childhood. They can be broadly divided into two categories reviewed below: intrinsic/individual factors and extrinsic/social or environmental factors (see Fig. 2).

Figure 2: Typology of factors influencing food neophobia

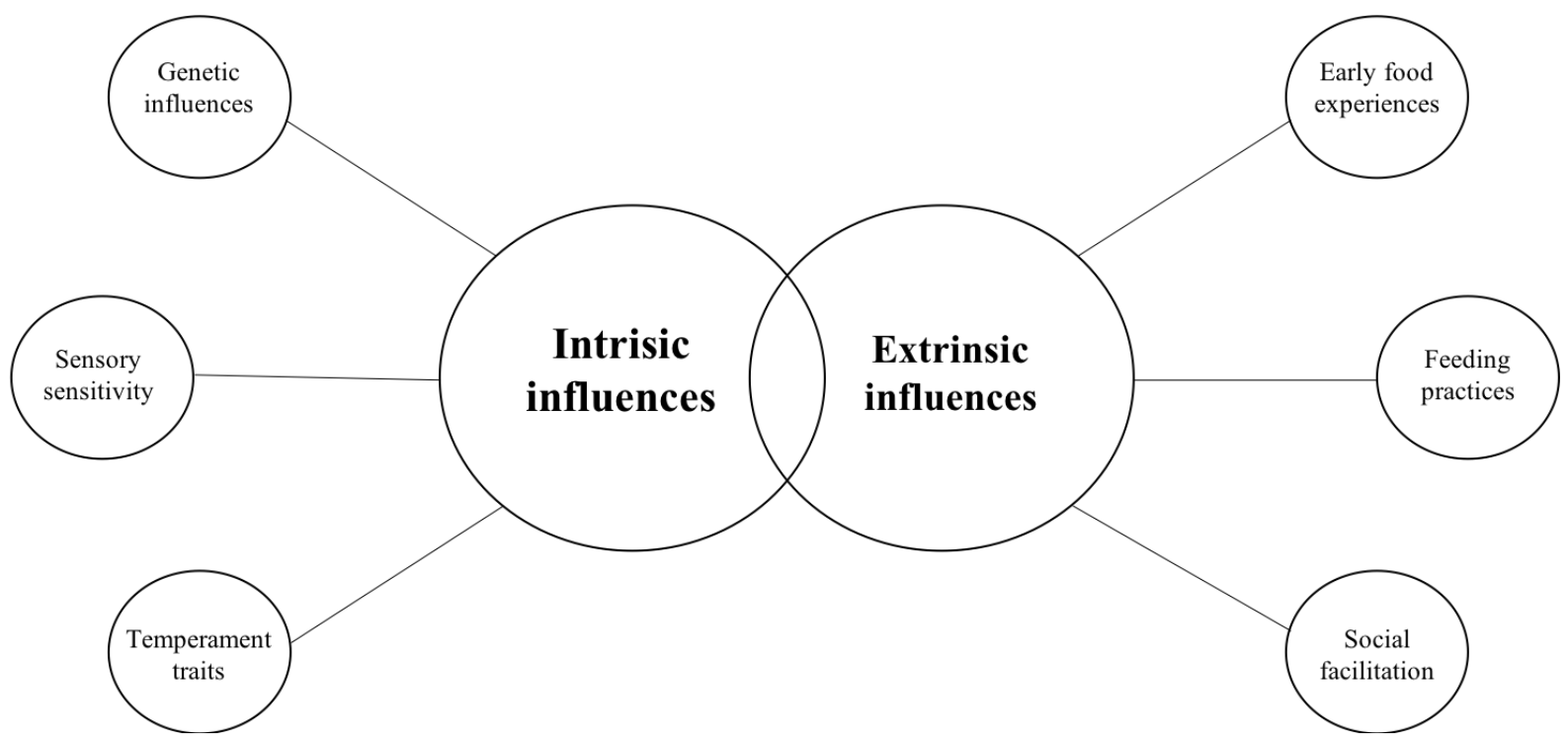




\subsection{Intrinsic influences on food neophobia}

\subsubsection{Genetic influences}

There is evidence that points to genetic influences underlying food neophobia. Studies to establish the contribution of genetic factors to food neophobia are of one main type: twin studies (see Cooke 2018 for a review). Twin studies compare similarity between monozygotic twins who share $100 \%$ of their genes and dizygotic twins who share $50 \%$ of their genes. These studies produce robust estimates of the contribution of genes on food neophobia (e.g., Cooke et al. 2007 ) and generally revealed that food neophobia is highly heritable (from $58 \%$ to $78 \%$ of the variance of food neophobia could be explain by genetic factors; Cooke 2018).

\subsubsection{The role of sensory sensitivity}

The anticipated sensory characteristics of foods (e.g., smell, texture, etc.) are recognized as a key determinant of decisions about whether to accept or reject a food (Farrow \& Coulthard 2018; Wadhera \& Capaldi-Phillips 2014), and evidence suggests that there is individual variety in how children perceive and evaluate sensory information, influencing food neophobia. Indeed, it has been shown that sensorily hypersensitive children are more likely to be more neophobichypersensitive children are more able to identify subtle changes in the sensory properties of stimuli, such as changes in smell, for instance (Blissett \& Fogel 2013; Farrow \& Coulthard 2018). Monnery-Patris and colleagues (2015) have explored smell and taste sensitivity in children by asking them to taste different solutions representing basic tastes, such as sweet or salty, and sniff different solutions representing pleasant or unpleasant odors, and found that food neophobia in toddlers was correlated with smell sensitivity, but not taste sensitivity. It has also been shown that neophobic children sniff new food from a further distance compared to their non- neophobic counterparts (Farrow \& Coulthard 2018). Other studies have also revealed an association between tactile sensitivity and food neophobia (Smith et al. 2005). For instance, it has been shown that tactile defensiveness (overreactions to the experiences of touch, or withdrawal responses to some typically inoffensive tactile stimuli perceived as offensive) is associated with high food neophobia (Smith et al. 2005). Together these findings indicate that neophobic children may experience exaggerated reactivity to olfactory and tactile food cues. It is interesting to note that these cues can be perceived before the food stimuli is tasted or swallowed, which is consistent with the claim that food neophobia serves a protective function (Rozin, 1979).

\subsubsection{Relationship between food neophobia and temperament traits}


Temperament traits are defined as individual differences in emotional and behavioral reactivity, that are relatively stable (Nicklaus \& Monnery-Patris 2018). Several temperament traits have been found to be associated with food neophobia (see Lafraire et al. 2016 and Nicklaus \& Monnery-Patris 2018 for reviews). It is assumed for instance, that food neophobia is associated with increased anxiety in children (Lafraire et al. 2016; Nicklaus \& Monnery-Patris 2018). More precisely, it has been shown that girls with food neophobia are more anxious than their non-neophobic counterparts. Sensation seeking has also been found to be negatively associated with food neophobia (Nicklaus \& Monnery-Patris 2018). Children who are greater sensation seekers, that is to say require a lot of stimulation to reach the appropriate level of awakening, tend to be less neophobic. Some researchers have also proposed that food neophobia could be a direct consequence of one specific dimension of temperament: approach/withdrawal (Moding \& Stifter 2016). This dimension characterizes individual differences in responses to new stimuli, such as toys, people or situations. Children who are low in approach tend to show negative affect toward new stimuli and withdraw from them (Moding \& Stifter 2016). In their longitudinal study, Moding and Stifter (2016) showed that children with low approach tendencies at 18 months had higher levels of food neophobia at 4.5 years of age. This set of studies raise the question of the selectivity of neophobia and suggest that food neophobic children are likely to present neophobic behavior in other domains as well (as revealed for instance in Moding and Stifter 2016).

\subsection{Extrinsic influences on food neophobia}

\subsubsection{Early food experiences and feeding practices}

Several studies have proposed that children's immediate environment is important in shaping their food neophobia (prenatal food experiences and feeding practices). Regarding prenatal and early experiences, evidence suggests that children are more willing to taste new flavors if they have experienced them through amniotic fluid (Cooke 2018; Mennella et al. 2001). Indeed, infants show less negative facial expressions to carrots when introduced to the diet if their mother had drunk carrot juice during the third trimester of pregnancy (Mennella et al. 2001). Regarding the influence of children's post-birth environment, certain feeding practices significantly affect food neophobia expression (Cooke 2018; Lafraire et al. 2016). For instance, commonly used parental feeding strategies such as food rewards, or pressure to eat, increase the expression of children's food neophobia (DeCosta et al. 2017; Nicklaus \& Monnery-Patris 2018; Lafraire et al. 2016). On the other hand, breast-feeding and offering children a high variety of vegetables at weaning seem to have a positive impact on food neophobia (Cooke 
2018; Nicklaus \& Monnery-Patris 2018). It is nevertheless important to note that most studies on food neophobia are not longitudinal (children are not followed during several months or years and are instead seen only once), therefore it is difficult to know the causality of the effects (is it because a child is neophobic that caregivers use more negative feeding practices or the inverse?).

\subsubsection{Social facilitation}

Another far and wide studied extrinsic factor influencing food neophobia is social facilitation (Lafraire et al. 2016; Nicklaus \& Monnery-Patris 2018), defined as an increase in the probability of performing a class of behavior in the presence of conspecifics performing the same class of behavior at the same time (see Herman 2015 and Higgs this volume, for a review of the food social facilitation effect). Put differently, social facilitation means that when a child eats in the presence of others eating food, her behavior is socially facilitated toward food intake. It has been shown for instance that toddlers are more willing to taste a new food if they see an adult eat it first (Addessi et al. 2005). Adults can serve as models for food acceptance e.g. an encouraging teacher or a friendly visitor in a classroom can foster food acceptance in young children. However social facilitation with peers (i.e., other children) seems more effective (Lafraire et al. 2016). Social facilitation is enhanced when models are familiar to children (Harper \& Sanders 1975) and are "prosocial" (i.e., nice and helping) (Hamlin \& Wynn 2012). Nevertheless, questions remain on what aspects of modeling is actually effective on children's food acceptance: the act of consuming the food, facial expressions or verbal messages that are associated with parental or peer consumption, or a combination of these factors (Blissett \& Fogel 2013).

\section{Interventions to overcome food neophobia}

Various reviews of existing interventions show that certain strategies efficiently decrease children's food neophobia (Appleton et al. 2016; DeCosta et al. 2017; Keller 2014; Knai et al. 2006). These interventions can generally be divided into two main types: interventions focusing on the eating context, and interventions focusing on the food itself (see Appleton et al. 2016 and DeCosta et al. 2017 for reviews). The interventions focusing on the eating context usually manipulate extrinsic factors known to influence neophobia, mentioned in the section above (see for instance Laureati et al. 2014, for an intervention in schools using peer social facilitation to 
decrease neophobia). Therefore, these interventions will not be further developed in this chapter (see DeCosta et al. 2017, for a review of the interventions focusing on the eating context).

Regarding the interventions based on the food itself, three types of interventions are widely used to decrease food neophobia: Flavor-Flavor Learning, Flavor-Nutrient-Learning and Mere Exposure (Appleton et al. 2016). 90\% of the interventions based on the food adopted at least one of these methods (Appleton et al. 2016). Flavor-Flavor-Learning (FFL) and FlavorNutrient-Learning (FNL) are two strategies based on conditioning: an association between a neutral conditioned stimulus and a positive unconditioned one will trigger a positive attitude toward the conditioned stimulus, even when the unconditioned stimulus is latter removed. The principle of FFL is that the pairing of a target food (often a new vegetable), with a taste that is already liked (e.g., a sweet or salty taste), should trigger positive attitude toward the target food. The principle of FNL is that the pairing of a target food, with a food with high energy density (e.g., enriched in fat), should trigger positive attitude toward the target food. Both FFL and FNL have been shown to positively enhance new fruit or vegetable acceptance in children (Appleton et al. 2016; Caton et al. 2013; Nicklaus \& Monnery-Patris 2018). For instance, Caton and colleagues (2013) found that infants accepted more plain artichoke puree at post-test, compared to baseline, when they had been offered ten times artichoke puree enriched in fat (FNL strategy). The third type of strategies used is Mere Exposure (also named Repeated Exposure). According to the Mere Exposure theory (ME), the exposure to one instance of a given food stimulus is sufficient to trigger a more positive attitude toward a subsequent instance of that particular stimulus, because it increases its familiarity (Zajonc, 1968). There is considerable support for the success of such ME on food acceptance in controlled experimental settings, as well as in more ecological settings like home or school environments (see Cooke 2007 for a review). For instance, some researchers asked caregivers to expose their 5- to 7-year-old children to bell peppers (an a priori new vegetable) for eight consecutive days, and found that, compared to a control group, children in the exposure group ate significantly more bell peppers at post-test than at baseline (Cooke 2007).

Despite the apparent efficiency of these three strategies (FFL, FNL, ME), it has been argued that conditions within studies are often confounded and that, in many studies that purported to measure conditioning effects (FFL and FNL interventions), exposure was actually not controlled for (Appleton et al. 2016). In fact, studies comparing directly these three strategies often found no advantage of conditioning (FFL and FNL) over mere exposure (ME) (Caton et 
al. 2013; Nicklaus \& Monnery-Patris 2018). For instance, Caton and colleagues (2013) found that infants who had been exposed to plain artichoke puree (ME condition) accepted plain artichoke puree as easily at post-test, as children who had been exposed to sweetened puree (FFL condition) or puree enriched in fat (FNL condition). As mere exposure interventions are easier to implement and seem to be as effective as FFL/FNL interventions, ME appears to be the simplest choice to enhance positive attitude towards fruits and vegetables and to decrease food neophobia (Appleton et al. 2016). It is nevertheless important to note that many exposure studies have failed to adopt an appropriate control group against which to compare the intervention group's changes in food attitudes (Appleton et al. 2016; Dazeley et al. 2012). For future investigations of $\mathrm{ME}$ it is important that control groups are closely matched to experimental groups in terms of demographics, but also in terms of the food tested at baseline and post-test (equivalence in familiarity, liking, energy density, food category, etc.).

\subsection{Taste exposure}

In the food domain, an exposure can occur in different modalities (taste, sight, smell etc.). The majority of studies on ME have only investigated whether taste exposure will be efficient to decrease food neophobia (see Cooke 2007 and Dazeley et al. 2012 for reviews). This central interest in taste exposure may be due to the seminal study of Birch and colleagues (1987). The authors directly compared the effect of visual and taste exposure on preschoolers liking for new fruits. They found that visual exposure led to an increase in liking for the appearance of the new fruits, while taste exposure led to an increase in liking for both the appearance and taste of the new fruits (Birch et al. 1987). Subsequent research on ME focused on taste exposure as it was thought to be necessary to enhance consumption of new foods like fruits and vegetables. Nevertheless, it could be argued that visual exposure would not likely directly influence taste preferences but can influence instead willingness to taste exposed foods (Dazeley et al. 2012).

While the benefits of repeated taste exposure are evident (Cooke 2007), these strategies may be difficult to implement outside the laboratory since 10 to 15 taste exposures to a new food item may be needed for its successful acceptance in preschool-aged children (Cooke 2007). This is a number greater than most parents are willing or able to provide (Dazeley et al. 2012). This number may even increase for highly neophobic children (De Wild et al. 2016) that yet need the most this kind of intervention.

\subsection{Visual exposure}


Because neophobic reactions result in foods being rejected on mere sight, before being tasted (Cashdan 1998; Dovey et al. 2008; Lafraire et al. 2016), it is reasonable to suppose that visual exposure could be as efficient to reduce food rejections than taste exposure. Indeed, according to some authors, a child will taste a food (e.g., a tomato) only if it visually matches him/her prototypical food representations in mind (e.g., the red and round tomato) (Dovey et al. 2008; Lafraire et al. 2016). Additionally, while after weaning infants are normally familiarized with the taste of several vegetables (through pureed baby foods), they often are not familiarized with the visual aspect of the whole vegetables. Furthermore, interventions seeking to enhance positive attitudes towards fruits and vegetables through taste exposure also increase the visual familiarity of the target. In studies that offer children repeated taste exposures, the food is both seen and tasted at each session, and it is difficult to isolate the effect of taste exposure alone (Dazeley et al. 2012). It is therefore plausible that the effectiveness of taste exposure interventions is partly due to a greater visual familiarity with the target food (Dazeley et al. 2012). Finally, from a practical point of view, it could also be less effort for caregivers to provide visual exposures to food (e.g., through picture books), especially if it occurs outside mealtimes, that carry the stress associated with ensuring that the child is consuming a healthy diet (Dazeley et al. 2012).

There is actually an encouraging body of evidence to support research into the impact of visual exposure on children food neophobia (see Dazeley et al. 2012 for a review). For example, some authors provided 2- to 6-year-old children with picture books about leeks or carrots. They showed that toddlers consumed more of the vegetable they had seen in their picture book (carrots or leeks), compared to a matched control vegetable. Importantly, some authors found similar changes in new vegetable acceptance for highly neophobic children and non neophobic children (Rioux et al. 2018). This suggests that visual exposure might be employed successfully with children for whom taste exposure has been showed to be less efficient (De Wild et al. 2016). Furthermore, Osborne and Forestell (2012), one of the few studies which directly compared visual and taste exposure since Birch and colleagues (1987), found that visual exposure was as efficient as taste exposure to enhance willingness to taste new fruits in 4- to 8year-old children. Interventions that use a more direct contact with unfamiliar food and enable children to play with it (so as children are not just passively exposed to pictures) also show promising results (Coulthard \& Ahmed 2017; Sapere Method, Nicklaus \& Monnery-Patris 2018). For instance, Coulthard and Ahmed (2017) found that children who played a game of bingo with real fruits and vegetables consume more of a new fruit than children who played the 
same game with picture stimuli. Additionally, they found that children who played the bingo game with pictures ate more of a new fruit after the session than children who simply viewed and sorted the same pictures (Coulthard \& Ahmed 2017). The potential for visual exposure to enhance children's willingness to taste new foods therefore deserves further exploration, especially to investigate whether it produces long term effects. Indeed, there is a dearth of research evidence supporting long term changes in children's diets after taking part in exposure interventions (Appelton et al. 206; Knai et al. 2006).

\section{3. The mechanisms behind Mere Exposure}

While a large body of research has investigated the potential effect of mere exposure, there is no consensus on its mechanistic explanation. It is yet important to understand the mechanisms behind such a strategy if we want to create the most possible effective interventions to decrease neophobia. One of the mechanisms by which exposure is assumed to engender a positive attitude toward a stimulus is thought to be "learned safety" (Cooke 2007): repeated ingestion of an unfamiliar food without negative post-ingestion consequences (e.g., nausea, illness) will lead to increased acceptance of this food (Cooke 2007). Nevertheless, the recent evidence that mere visual exposure could also enhance the acceptance of unfamiliar food items casts doubt on whether the "learned safety" hypothesis entirely explains the positive effect of exposure. There is an alternative explanation, which embodies a cognitive approach to the mere exposure effect (Aldrige et al. 2009; Lafraire et al. 2016). By increasing the amount of experience an individual has with any stimulus, repeated exposure increases the ease and speed with which the stimulus is categorized (Aldrige et al. 2009): when a food item is first presented to a child it is organized into categories relating to its characteristics (Murphy 2002). Knowledge gained through this first encounter allows for easier and faster categorization, when subsequently presented with the same or a similar food item (Aldridge et al. 2009). This gained ease in categorization in then hypothesized to lead to a positive attitude toward the stimulus. A recent study partly supports this hypothesis. Using a sorting task involving pictures of fruits and vegetables to assess categorization performances, Rioux and colleagues (2018) showed that categorization performance was associated with food neophobia disposition. Moreover, in the same study the authors found that a visual exposure intervention (table mats with pictures of new vegetables were displayed on school canteen tables for several days) was more efficient for those children with good categorization performance (Rioux et al. 2018). Efficiency of the intervention was measured via Willingness to Try New Vegetable tasks proposed at baseline and after the intervention (see section 2.3.2). Therefore, it seems that an ease in categorization 
indeed leads to more positive attitude toward exposed stimuli. Nevertheless, it remains to be seen if visual exposure intervention actually increases food categorization performances. There are few hints to think it could be the case. For instance, it has been recently shown that a short exposure to few animals through a class trip to a zoo enhances animal category-based induction performances (Badger \& Shapiro 2019).

\section{Summary and further directions}

Children moving into the second year of life have to make quite complex decisions about the foods they will eat and accept. Food neophobia, reviewed in the present chapter, is one of the strategies infants and children employ to deal with this complex problem of food selection. There has been considerable research on the topic, but some questions remain. First, there is a lack of cross-cultural studies to investigate the influence of culture on children's food neophobia. Most studies on the topic have been conducted in Western and rich countries (Nicklaus \& Monnery-Patris 2018). It will be valuable to investigate whether food neophobia is universally observed, and especially investigate children's neophobia in rural communities practicing more traditional subsistence. This investigation will first require to have tools to measure neophobia validated in several cultures (not merely translated), which is rarely done. Second, longitudinal studies on food neophobia are scarce and are much needed if we want to have a clear picture of its developmental pattern. The evidence provided by the rare longitudinal studies reveals that precursors of food neophobia are already present in infancy and contrasts with the common picture that neophobia arises as toddler become mobile (Dovey et al. 2018). Third, further studies could systematically evaluate the effectiveness of interventions aimed at decreasing food neophobia, investigate the mechanisms behind positive effects and test for long term effects (Appelton et al. 2016). Systematic comparison between interventions can prove difficult because a wide variety of outcome measures are used to assess efficacy (e.g., trait neophobia, willingness to try exposed foods, food consumption in grams), but is of interest to identify those of greatest benefit.

\section{References}

Addessi, E., Galloway, A. T., Visalberghi, E., \& Birch, L. L. (2005). Specific social influences on the acceptance of novel foods in 2-5-year-old children. Appetite, 45(3), 264-271. https://doi.org/10.1016/j.appet.2005.07.007 
Aldridge, V., Dovey, T. M., \& Halford, J. C. G. (2009). The role of familiarity in dietary development. $\quad$ Developmental Review, 29(1), 32-44. https://doi.org/10.1016/j.dr.2008.11.001

Alley, T. R. (2018). Conceptualization and measurement of human food neophobia. In Food Neophobia (pp. 169-192). Elsevier. https://doi.org/10.1016/B978-0-08-101931-3.000094

Appleton, K. M., Hemingway, A., Saulais, L., Dinnella, C., Monteleone, E., Depezay, L., ... Hartwell, H. (2016). Increasing vegetable intakes: rationale and systematic review of published interventions. European Journal of Nutrition, 55(3), 869-896. https://doi.org/10.1007/s00394-015-1130-8

Badger, J. R., \& Shapiro, L. R. (2019).We're going to the zoo: Interactive educational activities with animals boost category-based induction in children. Cognitive Development, 49, 110. https://doi.org/10.1016/j.cogdev.2018.10.003

Barends, C., de Vries, J. H. M., Mojet, J., \& de Graaf, C. (2014). Effects of starting weaning exclusively with vegetables on vegetable intake at the age of 12 and 23 months. Appetite, 81, 193-199. https://doi.org/10.1016/j.appet.2014.06.023

Birch, L. L., McPhee, L., Shoba, B. C., Pirok, E., \& Steinberg, L. (1987). What kind of exposure reduces children's food neophobia?: Looking vs. tasting. Appetite, 9(3), 171-178.

Blissett, J., \& Fogel, A., 2013. Intrinsic and extrinsic influences on children's acceptance of $\begin{array}{lllllll}\text { new foods. Physiology } \quad \& \quad \text { Behavior. } & 121, & 89 & 95 .\end{array}$ https://doi.org/10.1016/j.physbeh.2013.02.013

Cashdan, E. (1994). A sensitive period for learning. Human Nature, 5(3), 279-291. https://doi.org/10.1007/BF02692155

Cashdan, E. (1998). Adaptiveness of food learning and food aversions in children. Social Science Information, 37(4), 613-632.

Caton, S. J., Ahern, S. M., Remy, E., Nicklaus, S., Blundell, P., \& Hetherington, M. M. (2013). Repetition counts: repeated exposure increases intake of a novel vegetable in UK preschool children compared to flavour-flavour and flavour-nutrient learning. British Journal of Nutrition, 109(11), 2089-2097. https://doi.org/10.1017/S0007114512004126

Cole, N. C., An, R., Lee, S.-Y., \& Donovan, S. M. (2017). Correlates of picky eating and food neophobia in young children: a systematic review and meta-analysis. Nutrition Reviews, 75(7), 516-532. https://doi.org/10.1093/nutrit/nux024

Cooke, L. (2007). The importance of exposure for healthy eating in childhood: a review. Journal of Human Nutrition and Dietetics, 20(4), 294-301. 
Cooke, L. (2018). Genetic and environmental influences on food neophobia. In Food Neophobia (pp. 237-254). Elsevier. https://doi.org/10.1016/B978-0-08-101931-3.000124

Cooke, L. J., Haworth, C. M., \& Wardle, J. (2007). Genetic and environmental influences on children's food neophobia-. The American Journal of Clinical Nutrition, 86(2), 428-433.

Cooke, L., Carnell, S., Wardle, J. (2006). Food neophobia and mealtime food consumption in 4-5 year old children. International Journal of Behavioral Nutrition and Physical Activity, 3, 14. https://doi.org/10.1186/1479-5868-3-14

Cooke, L. J., \& Wardle, J. (2005). Age and gender differences in children's food preferences. British Journal of Nutrition, 93(5), 741. https://doi.org/10.1079/BJN20051389

Cooke, L., Wardle, J., Gibson, E.L. (2003). Relationship between parental report of food neophobia and everyday food consumption in 2-6-year-old children. Appetite, 41, 205206. http://dx.doi.org/10.1016/S0195-6663(03)00048-5

Coulthard, H., \& Ahmed, S. (2017). Non taste exposure techniques to increase fruit and vegetable acceptance in children: Effects of task and stimulus type. Food Quality and Preference, 61, 50-54. https://doi.org/10.1016/j.foodqual.2017.04.012

Damsbo-Svendsen, M., Frøst, M. B., \& Olsen, A. (2017). A review of instruments developed to measure food neophobia. Appetite, 113, 358-367. https://doi.org/10.1016/j.appet.2017.02.032

Dazeley, P., Houston-Price, C., \& Hill, C. (2012). Should healthy eating programmes incorporate interaction with foods in different sensory modalities? A review of the evidence. British Journal of Nutrition, 108(5), 769-777. https://doi.org/10.1017/S0007114511007343

de Lauzon-Guillain, B., Oliveira, A., Charles, M. A., Grammatikaki, E., Jones, L., Rigal, N., ... Monnery-Patris, S. (2012). A Review of methods to assess pparental feeding practices and preschool children's eating behavior: the need for further development of tools. Journal of the Academy of Nutrition and Dietetics, 112(10), 1578-1602.e8. https://doi.org/10.1016/j.jand.2012.06.356

de Wild, V. W. T., de Graaf, C., \& Jager, G. (2017). Use of different vegetable products to increase preschool-aged children's preference for and intake of a target vegetable: a randomized controlled trial. Journal of the Academy of Nutrition and Dietetics, 117(6), 859-866. https://doi.org/10.1016/j.jand.2016.11.006 
DeCosta, P., Møller, P., Frøst, M. B., \& Olsen, A. (2017). Changing children's eating behaviour - A review of experimental research. Appetite, 113, 327-357. https://doi.org/10.1016/j.appet.2017.03.004

Dovey, T. M., Staples, P. A., Gibson, E. L., \& Halford, J. C. G. (2008). Food neophobia and "picky/fussy" eating in children: A review. Appetite, 50(2-3), 181-193. https://doi.org/10.1016/j.appet.2007.09.009

Farrow, C., \& Coulthard, H. (2018). Multisensory evaluation and the neophobic food response. In Food Neophobia (pp. 219-236). Elsevier. https://doi.org/10.1016/B978-0-08-101931$3.00011-2$

Galloway, A.T., Lee, Y., Birch, L.L. (2003). Predictors and consequences of food neophobia and pickiness in young girls. Journal of the American Dietetic Association, 103, 692698. https://doi.org/10.1053/jada.2003.50134

Hamlin, J. K., \& Wynn, K. (2012). Who knows what's good to eat? Infants fail to match the food preferences of antisocial others. Cognitive Development, 27(3), 227-239. https://doi.org/10.1016/j.cogdev.2012.05.005

Harper, L. V., \& Sanders, K. M. (1975). The effect of adult's eating on young children's acceptance of unfamiliar foods. Journal of Experimental Child Psychology, 20, 203-214.

Harris, G. (2018). Food neophobia: Behavioral and biological influences. In Food Neophobia (pp. 193-217). Elsevier. https://doi.org/10.1016/B978-0-08-101931-3.00010-0

Helland, S.H., Bere, E., Bjornara, H.B., Overby, N.C. (2017). Food neophobia and its association with intake of fish and other selected foods in a Norwegian sample of toddlers: a $\begin{array}{lccccc}\text { cross-sectional } & \text { study. } & \text { Appetite, } & 114, & 110 & 117 .\end{array}$ https://doi.org/10.1016/j.appet.2017.03.025

Henriques, A. S., King, S. C., \& Meiselman, H. L. (2009). Consumer segmentation based on food neophobia and its application to product development. Food Quality and Preference, 20(2), 83-91. https://doi.org/10.1016/j.foodqual.2008.01.003

Herman, C. P. (2015) The social facilitation of eating. A review. Appetite, 86, 61-73. https://doi.org/10.1016/j.appet.2014.09.016

Keller, K. L. (2014). The use of repeated exposure and associative conditioning to increase vegetable acceptance in children: explaining the variability across studies. Journal of the Academy of Nutrition and Dietetics, 114(8), 1169-1173. https://doi.org/10.1016/j.jand.2014.04.016 
Klarer, M., Arnold, M., Gunther, L., Winter, C., Langhans, W., \& Meyer, U. (2014). Gut vagal afferents differentially modulate innate anxiety and learned fear. Journal of Neuroscience, 34(21), 7067-7076. https://doi.org/10.1523/JNEUROSCI.0252-14.2014

Knai, C., Pomerleau, J., Lock, K., \& McKee, M. (2006). Getting children to eat more fruit and vegetables: A systematic review. Preventive Medicine, 42(2), 85-95. https://doi.org/10.1016/j.ypmed.2005.11.012

Koivisto-Hursti, U.-K., \& Sjöden, P. (1996). Food and general neophobia in Swedish families: parent-child comparisons and relationships with serving specific foods. Appetite, 26, 107118. https://doi.org/10.1006/appe.1996.0009

Kral, T. V. E. (2018). Food neophobia and its association with diet quality and weight status in children. In Food Neophobia (pp. 287-303). Elsevier. https://doi.org/10.1016/B978-008-101931-3.00014-8

Lafraire, J., Rioux, C., Giboreau, A., \& Picard, D. (2016). Food rejections in children: Cognitive and social/environmental factors involved in food neophobia and picky/fussy eating behavior. Appetite, 96, 347-357. https://doi.org/10.1016/j.appet.2015.09.008

Laureati, M., Bertoli, S., Bergamaschi, V., Leone, A., Lewandowski, L., Giussani, B., ... Pagliarini, E. (2015). Food neophobia and liking for fruits and vegetables are not related to Italian children's overweight. Food Quality and Preference, 40, 125-131. https://doi.org/10.1016/j.foodqual.2014.09.008

Laureati, M., Bergamaschi, V., \& Pagliarini, E. (2014). School-based intervention with children. Peer-modeling, reward and repeated exposure reduce food neophobia and increase liking of fruits and vegetables. Appetite, 83, 26-32. https://doi.org/10.1016/j.appet.2014.07.031

Maratos, F. A., \& Sharpe, E. E. (2018). The origins of disordered eating and childhood food neophobia. In Food Neophobia (pp. 305-328). Elsevier. https://doi.org/10.1016/B978-008-101931-3.00015-X

Maiz, E., Balluerka, N. (2016). Nutritional status and Mediterranean diet quality among Spanish children and adolescents with food neophobia. Food Quality and Preference, 52, 133142. https://doi.org/10.1016/j.foodqual.2016.04.011

Mennella, J. A., Jagnow, C. P., \& Beauchamp, G. K. (2001). Prenatal and postnatal flavor learning by human infants. Pediatrics, 107(6), e88-e88. 
Moding, K. J., \& Stifter, C. A. (2018). Does temperament underlie infant novel food responses?: Continuity of approach-withdrawal from 6 to 18 Months. Child Development, 89(4), e444-e458. https://doi.org/10.1111/cdev.12902

Monnery-Patris, S., Wagner, S., Rigal, N., Schwartz, C., Chabanet, C., Issanchou, S., \& Nicklaus, S. (2015). Smell differential reactivity, but not taste differential reactivity, is related to food neophobia in toddlers. Appetite, 95, 303-309. https://doi.org/10.1016/j.appet.2015.07.021

Murphy, G. L. (2002). The big book of concepts. Cambridge, Mass: MIT Press.

Mumford, S. (1998). Dispositions. Oxford: Oxford University Press.

Nicklaus, S., Boggio, V., Chabanet, C., \& Issanchou, S. (2005). A prospective study of food variety seeking in childhood, adolescence and early adult life. Appetite, 44(3), 289-297. https://doi.org/10.1016/j.appet.2005.01.006

Nicklaus, S., \& Monnery-Patris, S. (2018). Food neophobia in children and its relationships with parental feeding practices/style. In Food Neophobia (pp. 255-286). Elsevier. https://doi.org/10.1016/B978-0-08-101931-3.00013-6

Osborne, C. L., \& Forestell, C. A. (2012). Increasing children's consumption of fruit and vegetables: Does the type of exposure matter? Physiology \& Behavior, 106(3), 362-368. https://doi.org/10.1016/j.physbeh.2012.01.006

Perry, R.A., Mallan, K.M., Koo, J., Mauch, C.E., Daniels, L.A., Magarey, A.M. (2015). Food neophobia and its association with diet quality and weight in children aged 24 months: a cross sectional study. International Journal of Behavioral Nutrition and Physical Activity, 12. https://doi.org/10.1186/s12966-015-0184-6

Pliner, P. (1994). Development of measures of food neophobia in children. Appetite, 23, 147163.

Pliner, P., \& Hobden, K. (1992). Development of a scale to measure the trait of food neophobia in humans. Appetite, 19(2), 105-120.

Rigal, N., Chabanet, C., Issanchou, S., \& Monnery-Patris, S. (2012). Links between maternal feeding practices and children's eating difficulties. Validation of French tools. Appetite, 58(2), 629-637. DOI: 10.1016/j.appet.2011.12.016

Rioux, C., Lafraire, J., \& Picard, D. (2017a). The Child Food Rejection Scale: Development and validation of a new scale to assess food neophobia and pickiness among 2- to 7-yearold French children. Revue Européenne de Psychologie Appliquée/European Review of Applied Psychology, 67(2), 67-77. https://doi.org/10.1016/j.erap.2017.01.003. 
Rioux, C., Lafraire, J., \& Picard, D. (2017). Food rejection and the development of food category-based induction in 2-6 years old children. Journal of Cognitive Psychology, 30(1), 5-17. https://doi.org/10.1080/20445911.2017.1367688

Rioux, C., Lafraire, J., \& Picard, D. (2018). Visual exposure and categorization performance positively influence 3- to 6-year-old children's willingness to taste unfamiliar vegetables. Appetite, 120, 32-42. https://doi.org/10.1016/j.appet.2017.08.016

Rioux, C., Picard, D., \& Lafraire, J. (2016). Food rejection and the development of food categorization in young children. Cognitive Development, 40, 163-177. https://doi.org/10.1016/j.cogdev.2016.09.003

Rozin, P. (1979). Preference and affect in food selection. In J. H. A. Kroeze (Ed.), Preference, Behaviour \& Chemoreception (pp. 289-297). London: Information Retrieval Limited.

Smith, A. M., Roux, S., Naidoo, N. T. (Raj), \& Venter, D. J. L. (2005). Food choices of tactile defensive children. Nutrition, 21(1), 14-19. https://doi.org/10.1016/j.nut.2004.09.004

Wadhera, D., \& Capaldi-Phillips, E. D. (2014). A review of visual cues associated with food on food acceptance and consumption. Eating Behaviors, 15(1), 132-143. https://doi.org/10.1016/j.eatbeh.2013.11.003

Wertz, A. E., \& Wynn, K. (2014). Thyme to touch: Infants possess strategies that protect them from dangers posed by plants. Cognition, 130(1), 44-49. https://doi.org/10.1016/j.cognition.2013.09.002

Włodarczyk, A., Elsner, C., Schmitterer, A., \& Wertz, A. E. (2018). Every rose has its thorn: Infants' responses to pointed shapes in naturalistic contexts. Evolution and Human Behavior, 39, 583- 593. https://doi.org/10.1016/j.evolhumbehav.2018.06.001

Zajonc, R. B. (1968). Attitudinal effects of mere exposure. Journal of Personality and Social Psychology, 9(2p2), 1. 